\title{
The context for the thematic grouping of rare diseases to facilitate the establishment of European Reference Networks
}

\author{
Teresinha Evangelista ${ }^{1 *}$, Victoria Hedley ${ }^{1}$, Antonio Atalaia ${ }^{1}$, Matt Johnson², Stephen Lynn ${ }^{1}$, Yann Le Cam² \\ and Kate Bushby ${ }^{1}$
}

\begin{abstract}
Background: In the past few years there has been a political imperative driving the creation of European Reference Networks as these are considered a promising way to achieve equity in access to the most up to date medical care across Europe. The right to equity in the access to care was established by the directive of the European Parliament and of the Council on the application of patients' rights in cross-border healthcare. The particular situation for Rare Diseases whereby sharing of expertise can be regarded as especially valuable, as well as the work that is already in place in the networking of Rare Diseases experts means that Rare Diseases are considered excellent models for the development of European Reference Networks.
\end{abstract}

Discussion: To be effective, a Rare Disease network should be based on the common effort of different stakeholders and be built on what is present in the community. European Reference Networks are an excellent model to overcome some of the specificities of rare diseases: scarcity of patients, resources and expertise. European Reference Networks with broad scope will allow the rare disease community the possibility of reaching a larger number of patients and more diversified rare diseases. The practical value of grouping rare diseases in broad networks is well demonstrated in different grouping systems present in Europe (EURORDIS grouping of diseases, "Les filières de santé maladies rares", Orphanet classification and the UK Research Model).

Summary: In this paper the authors, partners of EUCERD Joint Action, address some of the questions that surround the establishment of European Reference Networks. We will focus on how Rare Diseases could be efficiently grouped in order to constitute European Reference Networks and how they might be structured to allow each and every disease to benefit from networking.

Keywords: Rare Diseases, European policies, European reference networks, Grouping of rare diseases

\section{Background}

Rare diseases (RD) are defined in the European Union as life-threatening or chronically debilitating conditions that affect less than 5 per 10000 people. It has been well established in the academic and patient literature that the small number of patients and the geographic dispersion is an obstacle to the diagnosis, access to care, research and improvement of medical expertise. For a number of years it has been advocated that

\footnotetext{
* Correspondence: teresinha.evangelista@ncl.ac.uk

${ }^{1}$ John Walton Muscular Dystrophy Research Centre and MRC Centre for Neuromuscular Diseases, Institute of Genetic Medicine, Newcastle University, Newcastle upon Tyne NE1 3BZ, UK

Full list of author information is available at the end of the article
}

linking experts and pooling resources through networks would enable to overcome these obstacles, and significant expertise in networking across different RD groups has been established as demonstrated by the Orphanet Report: Series on Research Infrastructures for Rare Diseases in Europe (http://www.orpha.net/orphacom/ cahiers/docs/GB/Research_Infrastructures_for_rare_diseases_in_Europe.pdf).

The European Union has tried to combat the lack of specific health policies for rare diseases in the different Member States, through the establishment of an overall strategy for Member States to support the equity in 
access to prevention, diagnosis and care for patients with a RD throughout the European Union.

In March 2011 the European Directive on the application of patient's rights in cross-border healthcare [1] was adopted and established a legal framework for cross-border healthcare within the European Union (EU). The directive clarifies the rules of access to healthcare in a EU country different from the country of origin of the patient. It also clarifies the rules of reimbursement. One of the aims of the directive is to promote cooperation between the health systems of Member States including the establishment of European Reference Networks (ERNs). A network is by definition an association of individuals sharing common interests and providing mutual support and information. Due to its nature networks have the potential to cover large territorial areas. When we transpose these definitions to the organization of medical care, ERNs are an excellent model to overcome some of the specific problems of rare diseases: scarcity of patients, resources and expertise.

The recent publication of Implementing and Delegated Acts by the European Commission (EC) provides a framework for the creation of ERNs [2, 3]. Although RD are well placed to benefit from these instruments, it should be emphasised that ERNs were not specifically conceived for RD. It is also of note that the themes to be covered by ERNs are not prescribed in these acts.

In 2013 a study of the European Observatory on Health Systems and Policies [4] proposed some fundamental questions that should be tackled in the development of reference networks: which medical conditions or interventions should be addressed; what are the drivers and motivations for their creation; which regulatory, administrative and financial procedures are needed; and finally what are the impact and challenges for developing ERNs at European level.

The EUCERD Joint Action (EJA) is, since March 2012, assisting the EC with the formulation and implementation of its activities in the field of RD. Meantime, extensive work has been carried out by the EJA partners (John Walton Muscular Dystrophy Research Centre at Newcastle University and the European Organisation for Rare Diseases (EURORDIS)) with the European Union Committee of Experts in Rare Diseases/Commission Expert Group on Rare Diseases (EUCERD/CEGRD) on the generation of recommendations for RD ERNs. An important aspect with such a heterogeneous group of diseases as "rare diseases" is how to group them adequately to allow efficient and equitable functioning of RD ERNs.

In this paper we have focused on the process by which a decision was reached and adopted by the CEGRD as to how we could efficiently group RD in order to support the constitution of well-functioning ERNs.

\section{Discussion}

\section{Healthcare networks}

A significant body of work has been conducted around RD ERNs and the likely benefits have been well documented [1, 5-7]. ERNs for RD can be established considering different aspects, such as: disease frequency and prevalence, cost-effectiveness, or the need to reach sufficient numbers of patients to increase expertise and deliver the best quality of care. However, independently of the established priority, a RD ERN to be effective should be based on the common effort of different stakeholders and build on what is already present in the community. The principles for the establishment of RD ERNs were enshrined in the EUCERD recommendations on this topic in January 2013 [8].

The concept behind the implementation of ERNs as a model for healthcare provision has clearly evolved from the pure aim to improve cost-effectiveness to encompass improving quality of care and equity in access to healthcare. This shift makes it relevant to consider that the priority around RD ERNs should be reaching sufficient numbers of patients to increase expertise and deliver the best quality of care. Therefore, one of the main challenges for the society in general and for the rare diseases stakeholders in particular is to determine how ERNs should be organized i.e. how many should be created and how should different diseases be grouped in order to achieve the above-mentioned priority.

\section{Why organize ERNs around groups of diseases?}

A network, in social sciences, is defined as an association of individuals sharing common interests and providing mutual support and information [9]. Networks have the potential to achieve a large coverage depending on the number of members involved. When we transpose these definitions to the organization of medical care, ERNs are an excellent model to overcome some of the specific problems of rare diseases: scarcity of patients, resources and expertise. ERNs with a broad scope will allow the rare disease community the possibility of reaching a larger number of patients and a more diverse range of rare diseases.

With respect to existing networks, ERN status will need to represent a clear added-value such as: easier adoption and spread of innovations in medical science and health technologies; facilitation of medical training; faster dissemination of agreed standards of care and general knowledge in the area of expertise; and increased profile and recognition of the participants. ERNs will attract the best experts and more patients and although they are supposed to facilitate crossborder health care it is expected that the pooling of resources and the e-Health solutions will decrease the burden of travelling for the patients. Another expected 
though not explicit benefit is that the Networks and their members will be in a better position to apply for research funding and further develop and accelerate basic and translational research.

Existing networks in the RD field have arisen "ad hoc" in response to specific funding calls either from DG Sanco (now Santé) or from DG research. The diseases covered by these networks are often of low/very low prevalence and the networks highly focussed. The aims of the networks are highly variable and their sustainability has been a major issue (Table 1). No mechanism has yet been in place to ensure that all or even a majority of $\mathrm{RD}$ patients might have access to a network for their particular disease. In the process of drafting and adopting the EUCERD recommendations on RD ERNs, this issue of inclusivity was debated in some detail. Patients' organisations such as EURORDIS strongly advocated that ERNs should be inclusive and should not be created for every single RD, on the grounds that this would leave many patients without an 'umbrella' ERN [10]. Creating 6-8000 individual ERNs (as many as the estimated number of rare diseases) would be impossible, and not desirable from a clinical perspective.
Following the publication of the delegating and implementing acts, further discussion within the CEGRD indicated that more guidance was needed on the grouping of diseases into 'families' that can be addressed by comprehensive ERNs as a realistic and constructive approach.

\section{Grouping exercise}

In order to be successful, ERNs need to take into consideration existing national practices and networking systems which entails both opportunities and challenges. Existing networks and national healthcare authorities need to be made aware of the economical, scientific and patient care benefits of joint versus single disease networks.

The EUCERD Recommendations on Rare Diseases European Reference Networks (RD ERNS), published on 31 January 2013, aimed at provide guidance to the Member States and the EC on the criteria needed to be fulfilled to establish RD ERNs [8]. These recommendations were generated taking on board the results achieved by pilot ERNs funded through DG Sanco or DG Research between 2008 and 2013.

Table 1 Existing networks/projects in the rare disease field supported by EU funding

\begin{tabular}{|c|c|}
\hline Group of diseases & Networks/Projects \\
\hline Rare cardiac diseases & CHD - Congenital Heart Defects \\
\hline Rare connective tissue and musculoskeletal diseases & $\begin{array}{l}\text { PRINTO - paediatric rheumatology international trials organisation } \\
\text { ESDN: European Skeletal Dysplasia Network }\end{array}$ \\
\hline Rare hereditary metabolic disorders & $\begin{array}{l}\text { E-IMD - European registry and network for Intoxication type Metabolic Diseases } \\
\text { EUROGLYCANET CDG: Congenital Disorders of Glycosylation }\end{array}$ \\
\hline Rare haematological diseases & $\begin{array}{l}\text { EPNET - European Porphyria Network } \\
\text { EN-RBD - Rare Bleeding DisordersPaediatric Hodgkin's lymphoma Network } \\
\text { ENERCA - European Network for Rare and Congenital Anaemias } \\
\text { EUHANET - Haemophilia and the rare congenital deficiencies of other coagulation factors }\end{array}$ \\
\hline Rare immunological and auto- inflammatory diseases & EURO-HISTIO-NET - A reference network for Langerhans cell histiocytosis and associated syndrome \\
\hline Rare cancers & $\begin{array}{l}\text { ExPO-r-NeT - European Expert Paediatric Oncology Reference Network for Diagnostics } \\
\text { and Treatment } \\
\text { RARECARENet - Information network on rare cancers }\end{array}$ \\
\hline Rare hepatic diseases & EUROWILSON: European network on Wilson disease \\
\hline Rare neurological diseases & $\begin{array}{l}\text { NEUROPED - European Network of Reference for Rare Paediatric Neurological Diseases } \\
\text { LEUKOTREAT: Leukodystrophies } \\
\text { EUROSCA: European integrated project on spinocerebellar ataxiasE-Pilepsy - Refractory Epilepsy }\end{array}$ \\
\hline Rare Neuromuscular diseases & $\begin{array}{l}\text { Care-NMD - Improving care for Duchenne muscular dystrophy } \\
\text { TREAT-NMD - Neuromuscular network }\end{array}$ \\
\hline Rare skin disorders & $\begin{array}{l}\text { TAG - Together Against Genodermatoses } \\
\text { GENESKIN: European network on rare genetic skin diseases }\end{array}$ \\
\hline Rare Pulmonary diseases & $\begin{array}{l}\text { ECORN CF - Expert Advice on Cystic Fibrosis } \\
\text { PAAIR - Patient Associations and Alpha1 International Registry } \\
\text { ENCE CF-LAM-LTX - European networks of centres of expertise for CF (Cystic Fibrosis), LAM } \\
\text { (Lymphangioleiomyomatosis), and LTX (Lung Transplantation) } \\
\text { EUROCARE CF - Cystic Fibrosis }\end{array}$ \\
\hline Rare malformations and developmental anomalies & $\begin{array}{l}\text { DYSCERNE - Rare Dysmorphic Syndromes } \\
\text { EUROCRAN - Craniofacial anomalies }\end{array}$ \\
\hline Rare Kidney diseases & EuroCYST initiative - Polycystic Kidney Diseases \\
\hline
\end{tabular}


After the publication of the Delegated and Implementing Acts in March 2014 the EJA conducted a mapping exercise to explore the areas where the previous recommendations needed further attention from the EC Expert Group on Rare Diseases. That mapping exercise was the basis for an addendum to the EUCERD recommendations. Based on the content of the EUCERD Recommendations and the content of the Acts the Addendum to the Recommendations suggested an illustrative grouping of $\mathrm{RD}$ as a rational approach to RD ERN planning and to ensure coverage of all RD.

We based our exercise about the grouping of rare diseases on several possible models such as: the alignment of diseases by clinical area (e.g. Neurology, Neuromuscular, Psychiatry, Skin, Kidney, etc.), by clinical group (e.g. Genetic Disorders, Metabolic Disorders, Epilepsy, Oncology), clinical intervention area (e.g. Transplantation, Gene Therapy, Radiotherapy), shared molecular aetiology [11] (e.g. Underlying disease mechanism or pathway such as fibrosis or inflammation) or mixed models.

Several successful examples of centres of expertise and international networks for RD already exist, which may be viewed as concrete, trusted 'solutions' in their field of expertise and should be approached as case-studies from which one can derive important lessons for future ERNs. These tend to be grouped by clinical area or clinical grouping (e.g. rare anaemias, neuromuscular diseases, metabolic diseases, rare epilepsies, etc.).

An ERN based on a specific treatment area, such as for example Gene Therapy, although attractive when considering clinical trials or therapy development would inevitably leave numerous rare disease patients without a "home". This model would obviously be involved with different clinical areas and could be regarded as transversal, cutting across numerous medical specialties. Such a transversal ERN, could not replace ERNs based on clinical areas or clinical groups and would have to coexist alongside these.

ERNs based on shared molecular mechanisms would, as the former model, be a way of addressing problems related with clinical trials and drug development. Drugs that target a molecular pathway that is common to multiple diseases can, in principle, be used to treat more than one disease. However, though the concept is interesting in particularly from a research perspective, such an ERN might prove rather restrictive and too "single issue", especially given the multidisciplinarity inherent in the expectations asked of an ERN.

We therefore compared five examples of disease grouping based on clinical areas/groups (the Classical Medical Ontology; the Orphanet classification; the EURORDIS preliminary proposal for grouping of rare diseases; the French filières; and the UK Research Model as established by the NIHR Translational Research Collaboration on
RD). This comparison enabled us to propose a preliminary strategy for grouping rare diseases based on the merging of the common items. The classical medical ontology is the one used in Internal Medicine textbooks and is usually organised according to major organs and systems. Although incomplete for $\mathrm{RD}$, it is widely used for teaching and is imbedded in the physicians' way of thinking. A well-known example is the classification used in Harrison's Principles of Internal Medicine [12].

The Orphanet classification of rare diseases is structured around 116 groups of diseases. For the purposes of creating ERNs, 116 'categories' would be excessive; however, in terms of thematic grouping Orphanet proposes 31 RD categories [http://www.orphadata.org/cgi-bin/inc/ product3.inc.php]. It is a useful scientific classification; however, when dealing with ERNs there are some overlaps and some redundant groups. If, for instance, we consider the broad group of "Rare genetic diseases" should sickle cell anaemia be in the scope and expertise of an ERN for rare genetic diseases or for rare haematological diseases? Is it practical to have an ERN devoted to rare intoxications defined by Orphanet as rare intoxications due to medical products, or should these be incorporated in ERNs dealing with, for instance cardiac disorders for digitalis intoxication or with oncology for cytostatic intoxication?

The EURORDIS proposal, presented at the workshop "Rare Disease European Reference Networks (RD ERNs) and the use of structural funds to support activities for RD" held in Rome on the 28-29th October 2014 organises ERNs by clinical area and was based upon research undertaken by EURORDIS collaborators. This was an outline document, kindly made accessible to us by EURORDIS, which drafted groupings to facilitate discussion with the Council of National Alliances, Council of European Federations and EURORDIS members (Table 2). The Second French National Plan for Rare Diseases mandated the creation of 'filières de santé maladies rares'. These "French national rare disease healthcare networks" aim to coordinate all the missions and activities of groups of centres of expertise and related competence centres in charge of coherent groups of related rare diseases. At present 23 official networks were created [http://www.sante.gouv.fr/les-filieres-desante-maladies-rares.html]. The 'Filières de santé maladies rares' were adequately based on what was already in place in the French health system. The main problem stays when we try to extrapolate these country-specific pathways to a broader coverage. Also, the 'themes' range from relatively broad (e.g. inborn metabolic diseases or developmental anomalies and malformations) to diseasespecific (e.g. Amyotrophic Lateral Sclerosis). Given the lack of therapeutic options for the vast majority of the 6-8000 rare diseases, there is a necessary link between 
Table 2 EURORDIS Proposal for grouping of diseases for ERNs

\begin{tabular}{l} 
1. Undiagnosed Conditions RD ERN \\
2. Immunologically-mediated and Systemic RD ERN \\
3. Cardio-Vascular Diseases RD ERN \\
4. Malformations/Medical Genetics/Neuropaediatrics RD ERN \\
5. Dermatological diseases RD ERN \\
6. Endocrinal Diseases RD ERN \\
7. Hepatic gastroenterological and Severe Intestinal Disorders RD ERN \\
8. Non-Malignant Haematological Diseases RD ERN \\
9. Hereditary Metabolic Diseases RD ERN \\
10. Neurological Diseases RD ERN \\
11. Neuromuscular RD ERN \\
12. Pulmonary RD ERN \\
13. Kidney RD ERN \\
14. Connective Tissue Framework and Specialist Rheumatology \\
Diseases RD ERN \\
15. Head \& Neck Malformations RD ERN and Sensory Diseases RD ERN \\
(including rare ophthalmological, congenital and genetic disease) \\
16. Cancers RD ERN \\
17. Other Rare Diseases RD ERN \\
18. Rare Orthopaedic diseases including Complex Spinal Disorders RD ERN \\
19. Women, neonatal and children RD ERN \\
\hline
\end{tabular}

research and clinical care provision; therefore, we have also considered in our analysis the example of how RD have been grouped by the UK National Institute for Health Research (NIHR) Translational Research Collaboration in Rare Diseases (Table 3).

Table 3 UK grouping of rare diseases for research purposes

1. Cancer
2. Cardiovascular
3. Dementia and Neurodegenerative
4. Eye Disease
5. Gastrointestinal
6. Immunological Disorders
7. Metabolism
8. Musculoskeletal Disorders
9. Neuromuscular Disorders
10. Non-Malignant haematology
11. Paediatric (cross-cutting)
12. Renal Disease
13. Respiratory Disease
14. Skin

Legend: example of how RD have been grouped by the UK National Institute for Health Research (NIHR) - Translational Research Collaboration in Rare Diseases
Table 4 ORPHANET Classifications

Rare cardiac diseases

Developmental anomalies during embryogenesis

Inborn errors of metabolism

Rare gastroenterological diseases

Rare neurological diseases

Rare abdominal surgical diseases

Rare hepatic diseases

Rare respiratory diseases

Rare urogenital diseases

Rare surgical thoracic diseases

Rare skin diseases

Rare renal diseases

Rare eye diseases

Rare endocrine diseases

Rare haematological diseases

Rare immunological diseases

Rare systemic and rheumatologic diseases

Rare odontological diseases

Rare circulatory system diseases

Rare bone diseases

Rare otorhinolaryngological diseases

Rare infertility disorders

Rare tumours

Rare infectious diseases

Rare intoxications

Rare gynaecological and obstetric diseases

Rare surgical maxillo-facial diseases

Rare allergic disease

Teratological disorders

Rare cardiac malformations

Rare genetic diseases

Legend: Each of these headings represents a separate and more exhaustive classification. (http://www.orphadata.org/cgi-bin/inc/product3.inc.php)

\section{Conclusion}

After analysing these different models we have tried to merge the common lines having in consideration that the grouping strategy has to incorporate the need of having healthcare providers with different roles/expertise in any network, the fact that a healthcare provider may take part in different ERNs, the need to have a significant collaboration across ERNs as well as within, and that fluidity and inter-communication between ERNs should be ingrained in the structure of the network. Another important question that was debated was whether a dedicated ERN for undiagnosed patients would be feasible or desirable. Here the distinction between truly undiagnosed patients (those in whom a precise diagnosis 
is not possible and who may require access to research programmes for a diagnosis to be achieved, such as is available via the NIH undiagnosed scheme in the USA) and the patients who are known to have a particular type of disease but not yet a precise diagnosis, needs to be made. As an alternative to a dedicated 'undiagnosed' RD ERN, it is suggested that each ERN should maintain a 'forum' for undiagnosed patients suspected to fall within their area of expertise. Considering what was stated about how RD ERNs should be structured, the necessity to encompass all rare disease patients and the merging of the different models we have studied, we suggest the constitution of 22 broad groups (Table 5). This list was posteriorly presented to the Commission Expert group on Rare Diseases for discussion. Some changes were suggested and the final grouping (Table 6) was published as part of the Addendum to the EUCERD recommendations of January 2013 [8].

As with the previously described models, the proposed model is not perfect. However some of the difficulties can be overcome as long as the ERNs are organized as flexible structures with capacity to adjust

Table 5 Grouping of RD for Future ERNs, based on areas of overlap in the systems outlined in the text

1. Rare cardiac diseases (with rare cardiac malformations ERN
included or separate)
2. Rare connective tissue and musculoskeletal diseases
3. Rare hereditary metabolic disorders
4. Rare haematological diseases
5. Rare diseases of brain development and rare intellectual disabilities
6. Rare auto-immune and auto inflammatory diseases
7. Rare cancers
8. Rare hepatic diseases
9. Rare gastrointestinal diseases
10. Rare neurological diseases
11. Rare neuromuscular diseases
12. Rare skin disorders
13. Rare pulmonary diseases
14. Rare malformations and developmental anomalies
15. Rare endocrine diseases
16. Rare urogenital diseases
17. Rare renal diseases
18. Rare multi-systemic vascular diseases
19. Rare head and neck diseases
20. Rare gynaecological and obstetric diseases
21. Rare eye diseases
22. Rare bone diseases

Legend: The present list was proposed by the EUCERD Joint Action team and was subsequently submitted to the Commission Expert group on Rare Diseases for discussion
Table 6 Grouping rare diseases in thematic networks

Rare immunological and auto-inflammatory diseases

Rare bone diseases

Rare cancers and tumours

Rare cardiac diseases

Rare connective tissue and musculoskeletal diseases

Rare malformations and developmental anomalies and rare intellectual disabilities

Rare endocrine diseases

Rare eye diseases

Rare gastrointestinal diseases

Rare gynaecological and obstetric diseases

Rare haematological diseases

Rare craniofacial anomalies and ENT (ear, nose and throat) disorders

Rare hepatic diseases

Rare hereditary metabolic disorders

Rare multi-systemic vascular diseases

Rare neurological diseases

Rare neuromuscular diseases

Rare pulmonary diseases

Rare renal diseases

Rare skin disorders

Rare urogenital diseases

Legend: Thematic grouping of networks published in RARE DISEASE

EUROPEAN REFERENCE NETWORKS: ADDENDUM TO EUCERD

RECOMMENDATIONS OF JANUARY 2013

to the reality and maintain enough plasticity to share patients amongst them, in particular the undiagnosed ones (Fig. 1).

\section{Summary}

ERNs must be sufficiently broad and flexible in their scope to adjust to real life. When establishing ERNs one should aim to encompass all rare disease patients including those without a precise diagnosis. To achieve these goals ERNs should not be considered as static, nonflexible structures. They should take under their umbrella those patients that, according to the state of the art, are considered undiagnosed but whose clinical manifestations fall in the respective area of expertise. ERNs should aim to interact with other ERNs, should share common ontologies and coding systems, and should have inter-operational IT technologies. Despite variation in the details of some of the models, a group of approximately 22-25 clinical disease areas form the core of the different systems and could be a starting point for a cohesive European approach.

As with social networks [9] a RD ERN should have a cooperative structure with decentralized authority. It has 


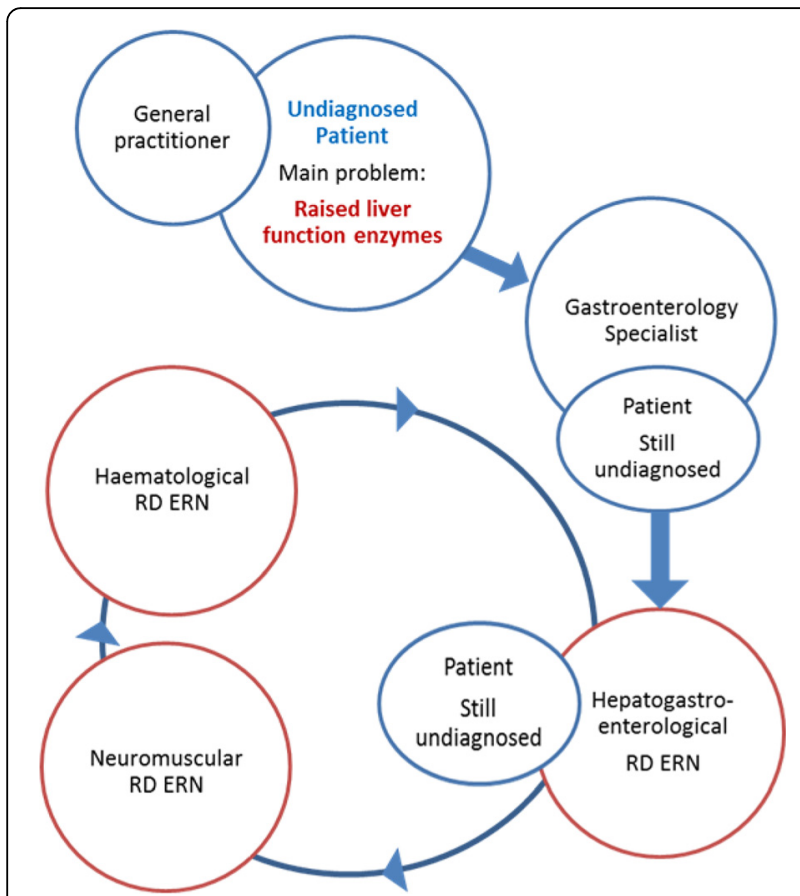

Fig. 1 Possible pathway for undiagnosed patients in the presence of flexible networks

to build on critical mass and to develop collective intelligence. Collective intelligence principles, a collective rather than an hierarchical approach to decision making and development of intellectual content, are not strange to the healthcare systems and have been seen as a way of maintaining access to services that otherwise due to the scarcity of resources the healthcare system would not be able to offer [13]. For RDs, ensuring the integration of these core features poses unique challenges, yet, simultaneously promises to generate unique added-value. For example, in terms of 'collective intelligence', RD ERNs will support expert collaboration and communication. It is also known that in the past a lack of 'critical mass' has hindered RD research and healthcare provision, ERNs will facilitate this by bringing together patients and healthcare providers in a sustainable structure with a clear position within healthcare policy.

\section{Abbreviations}

RD: Rare Diseases; EU: European Union; ERNs: European Reference Networks; EC: European Commission; EUCERD: European Union Committee of Experts in Rare Diseases; CEGRD: Commission Expert Group on Rare Diseases; EJA: EUCERD Joint Action; EURORDIS: European Organisation for Rare Diseases.

\section{Competing interests}

None of the authors have any competing interests.

\section{Authors' contributions}

TE conceived and participated in the design of the work and coordination and draft of the manuscript, is accountable for all aspects of the work in ensuring that questions related to the accuracy or integrity of any part of the work are appropriately investigated and resolved. VH participated in the design of the work and helped drafting and critically revising the manuscript. AA gave substantial contribution to the conception of the work, participated in its design and helped with the bibliographical support. MJ and YL contributed with bibliographical research, helped with the design of the study and revising the manuscript. SL helped revising the manuscript critically. KB give substantial contributions to the conception of the work and help revising it critically for important intellectual content is responsible for the final approval of the version to be published. All authors read and approved the final manuscript.

\section{Authors' information}

The authors are members of the EUCERD Joint Action and presently of RD-Action. $Y L$ and $K B$ are members of the Commission Expert Group on Rare Diseases. KB is a NIHR Senior Investigator.

\section{Acknowledgements}

This work was enabled by the EUCERD JOINT ACTION: WORKING FOR RARE DISEASES which has received funding from the European Union's Health Programme (2008-2013, contract no. 20112201)

The work was supported by the TREAT-NMD Alliance; the Medical Research Council (MRC) Centre for Neuromuscular Diseases (Newcastle and London) [Grant reference G1002274, grant ID 98482]. The National Health Service (NHS) and the National Institute for Health Research (NIHR). \#ls a NIHR Senior Investigator

\section{Author details}

'John Walton Muscular Dystrophy Research Centre and MRC Centre for Neuromuscular Diseases, Institute of Genetic Medicine, Newcastle University, Newcastle upon Tyne NE1 3BZ, UK. ${ }^{2}$ European Organisation for Rare Diseases (EURORDIS), Paris, France.

Received: 26 November 2015 Accepted: 4 February 2016

Published online: 24 February 2016

\section{References}

1. Directive 2011/24/EU of the European Parliament and of the Council of 9 March 2011 on the application of patients' rights in cross-border healthcare. OJ L 88, 4.4.2011, p. 45-65

2. 2014/286/EU: Commission Delegated Decision of 10 March 2014 setting out criteria and conditions that European Reference Networks and healthcare providers wishing to join a European Reference Network must fulfil Text with EEA relevance. OJ L 147, 17.5.2014, p. 71-78

3. 2014/287/EU: Commission Implementing Decision of 10 March 2014 setting out criteria for establishing and evaluating European Reference Networks and their Members and for facilitating the exchange of information and expertise on establishing and evaluating such Networks Text with EEA relevance. OJ L 147, 17.5.2014, p. 79-87

4. Palm W, Glinos IA, Rechel B, Garel P, Reinhard B, Figueras J. Building European Reference Networks in Health Care: Exploring concepts and national practices in the European Union. European Observatory on Health Systems and Policies; 2013. http://www.euro.who.int/_data/assets/pdf_file/ 0004/184738/e96805-final.pdf.

5. Position paper: EUROPEAN REFERENCE NETWORKS FOR RARE DISEASES EURORDIS; May 2012. http://www.eurordis.org/sites/default/files/EURORDIS_ Position_paper_on_ERN_May_2012.pdf

6. EUCERD Report: Preliminary analysis of the experiences and outcomes of pilot European Reference Networks for rare diseases. May 2011. http://www. eucerd.eu/?post_type $=$ document\&p $=1357$.

7. RDTF Report: European Reference Networks in the field of Rare Diseases: State of the art and Future Directions - July 2008. http://www.eucerd.eu/ ?post_type=document\&p=1204.

8. EUCERD Recommendations on Rare Diseases European Reference Networks (RD ERN) (31 January 2013) and Addendum (10 June 2015). http://www. eucerd.eu/?post_type $=$ document\& $=2207$.

9. Plastrik P and Taylor M. NET GAINS: A Handbook for Network Builders Seeking Social Change. Version 1.0 (2006). http://networkimpact.org/ downloads/NetGainsHandbookVersion1.pdf

10. Andersen T, Le Cam Y, Weinman A. European Reference Networks for rare diseases: the vision of patients. Blood Transfus. 2014;12 Suppl 3:5626-7. 
11. Brooks PJ, Tagle DA, Groft S. Expanding rare disease drug trials based on shared molecular aetiology. Nat Biotechnol. 2014;32:515-8.

12. Harrison's Principles of Internal Medicine, 18th Edition. Editors: Longo D L, Fauci A S, Kasper D L, Hauser S L, Jameson J L, Loscalzo J. Publishers: McGraw-Hill Medical

13. Collective Intelligence in Healthcare. http://www.oakleigh.co.uk.

Submit your next manuscript to BioMed Central and we will help you at every step:

- We accept pre-submission inquiries

- Our selector tool helps you to find the most relevant journal

- We provide round the clock customer support

- Convenient online submission

- Thorough peer review

- Inclusion in PubMed and all major indexing services

- Maximum visibility for your research

Submit your manuscript at www.biomedcentral.com/submit
Biomed Central 